\title{
Dynamic Range Consideration in MIMO Systems with Hybrid Antenna Selection
}

\author{
Javad Ahmadi-Shokouh \\ Antenna and Microwave Research Laboratory, University of Sistan and Baluchestan, Zahedan, Iran \\ Correspondence should be addressed to Javad Ahmadi-Shokouh, jahmadis@maxwell.uwaterloo.ca \\ Received 6 December 2010; Revised 13 May 2011; Accepted 22 June 2011 \\ Academic Editor: Ananda Sanagavarapu Mohan
}

Copyright ( $) 2011$ Javad Ahmadi-Shokouh. This is an open access article distributed under the Creative Commons Attribution License, which permits unrestricted use, distribution, and reproduction in any medium, provided the original work is properly cited.

\begin{abstract}
A Hybrid Antenna Selection (HAS), also called Soft Antenna Selection (SAS), method is basically implemented by a Linear Network (LN) located in RF domain of Multiple Input Multiple Output (MIMO) systems. In this paper, we evaluate the SASMIMO system, which is optimally tuned based on spatial multiplexing/diversity transmissions, in terms of receiver dynamic range issue. To this end, an SNR analysis is first performed for a reference point that is the input of Receiver Chain Block (RCB). Different systems are then compared based on a standard receiver, that is, WLAN $802.11 \mathrm{~b}$. A three Dimensional (3D) ray-tracing modeling is applied to assist this evaluation. The simulation results for a case study show that although the optimum post-LNA SAS works like a full-complexity MIMO in the spatial multiplexing/diversity transmission strategies, it provides even a better SNR to the baseband, that is, it reveals a receiver dynamic range improvement.
\end{abstract}

\section{Introduction}

The idea of exploiting controllable Radio Frequency (RF) preprocessing scheme, so-called Soft Antenna Selection (SAS), in Multiple Input Multiple Output (MIMO) systems is recently proposed in [1]. Due to the adaptive beamforming capabilities, the proposed SAS method has shown a significantly improved performance compared to the traditional antenna selection methods for MIMO systems [1]. It is shown in [1] that the optimum SAS method can even achieve the performance of a full-complexity MIMO system in which the receive antennas are directly connected to the $\mathrm{RF}$ chains and have the same number as the SAS receive antennas. In [1], the SAS structure is optimized based on the instantaneous channel states for either spatial multiplexing or diversity MIMO transmission. A similar RF preprocessing architecture is also used in [2]; however, in which the linear RF preprocessing matrix is tuned only based on the large-scale statistics of the channel. Moreover, an optimal SAS is found for an interference MIMO channel in [3]. Since an SAS method is essentially applied in RF domain the practical constraints of the implementation must be considered. Dynamic limits of the employed components are important factors which have to be applied in the RF domain processing. These factors show the acceptable upper and lower limits of the signal strength in the practical RF components. For instance, a signal detector does not recognize the received signals below a threshold. Obviously, a better dynamic range can increase the cost of the used devices. These factors are not considered in the approaches applied by the above-mentioned references. In fact, an exact noise analysis for the MIMO system equipped with the SAS module, called SAS-MIMO system for the rest of the paper, is needed to investigate on these limits.

Two types of the SAS-MIMO systems are introduced in the literature in terms of the implementation referenced to Low Noise Amplifier (LNA): post-LNA SAS [1] and pre-LNA SAS [4]. In the post-LNA SAS-MIMO system, the RF preprocessing, that is, SAS, is performed between LNA and Receiver Chain Block (RCB) while in the preLNA SAS architecture the SAS network is applied between the antenna elements and LNAs. It is shown in [5] that the post-LNA SAS-MIMO system outperforms the pre-LNA SAS in both spatial multiplexing and diversity transmission strategies and therefore we consider only the post-LNA SAS architecture in our dynamic range investigation. In this 
TABLE 1: SAS module components.

\begin{tabular}{lccc}
\hline Type & Frequency & Loss & Reference \\
\hline Phase shifter & $2-4 \mathrm{GHz}$ & $3.5 \mathrm{~dB}$ & {$[7]$} \\
Power splitter & $2-4 \mathrm{GHz}$ & $6+0.6 \mathrm{~dB}$ & {$[8]$} \\
Power combiner & $0.8-2.5 \mathrm{GHz}$ & $1 \mathrm{~dB}$ & {$[9]$} \\
\hline
\end{tabular}

regard, a rigorous Signal-to-Noise Ratio (SNR) analysis is performed for the SAS-MIMO as well as a reference single $\mathrm{RF}$ chain receiver used in common MIMO architectures. To evaluate the dynamic range improvement using a SASMIMO system, a ray-tracing channel simulation is also applied. The simulation results show that although the SAS method performance is generally said to be upper-bounded by a full-complexity MIMO, it shows a better signal coverage than the full-complexity MIMO.

The rest of the paper is organized as follows. In Section 2, a rigorous SNR analysis is done for post-LNA SAS-MIMO as well as a single RF chain receiver. Moreover, a deterministic ray-tracing simulation is used to realize a typical example set based on a 802.11 b wireless link in Section 3. Finally, Section 4 concludes the paper.

\section{SNR Analysis}

In this section, two difference receiver architectures are analyzed. (1) A single RF chain receiver as a reference. (2) SAS-MIMO receiver with active phased array, that is, postLNA SAS-MIMO. The SAS module in the latter is a phase only LN built by the component given in Table 1 . In this analysis, we assume that there is no dominant sky noise, and the antenna temperature is the same as room temperature $T_{A}=T_{0}$, for example, in terrestrial mobile communications [6, page 457].

2.1. Reference Single RF Channel Receiver. Consider a regular transceiver with single antenna connected to the RF chain depicted in Figure 1. Such a structure is used in common MIMO structures like a full-complexity MIMO system. Now, assuming that the receiver noise dominates, that is, no sky noise, the noise power at the RCB input is given by

$$
\begin{aligned}
N_{\text {Single }} & =\left(N_{A}+N_{\mathrm{TL}}\right) G_{\mathrm{TL}} G_{\mathrm{LNA}}+N_{\mathrm{LNA}} G_{\mathrm{LNA}} \\
& =F_{\mathrm{LNA}} G_{\mathrm{LNA}} K T_{0} B_{\omega},
\end{aligned}
$$

where $K$ and $B_{\omega}$ are Boltzman constant and frequency bandwidth, respectively. Moreover, $G_{\mathrm{TL}}<1, G_{\mathrm{LNA}}, F_{\mathrm{LNA}}$ represent the transmission line gain, the LNA gain and the LNA noise figure. In (1), $N_{A}, N_{\mathrm{TL}}$ and $N_{\mathrm{LNA}}$ are, respectively, the antenna noise, the noise introduced by the transmission line and the LNA noise:

$$
\begin{gathered}
N_{A}=K T_{A} B_{\omega}, \\
N_{\mathrm{TL}}=\left(\frac{1}{G_{\mathrm{TL}}}-1\right) K T_{0} B_{\omega}, \\
N_{\mathrm{LNA}}=\left(F_{\mathrm{LNA}}-1\right) K T_{0} B_{\omega} .
\end{gathered}
$$

Note that the introduced noise by each block is modeled at the input [10]. The signal power at the RCB input is also given by

$$
S_{\text {Single }}=|h \cdot s|^{2} G_{\mathrm{TL}} G_{\mathrm{LNA}} \text {, }
$$

where $h$ and $s$ are the channel and transmitted signal, respectively. Hence, the resulting $\mathrm{SNR}$, called $\mathrm{SNR}_{0}$, is given by

$$
\mathrm{SNR}_{0}=\frac{S_{\text {Single }}}{N_{\text {Single }}}=\frac{G_{\mathrm{TL}}|h \cdot s|^{2}}{F_{\mathrm{LNA}} K T_{0} B_{\omega}} .
$$

2.2. Active Phased Array Receiver. To calculate SNR for an SAS module with an active phased array, we consider one out of $M_{r}$ input receiver branches depicted in Figure 2. As mentioned before, in a perfect match condition the noise power coming from LNA is divided by $M_{r}$ but we only add up $1 / M_{s}$ noise power of input ports at the combiner output. As shown in the figure, the phase shifter has a gain $G_{\phi}$, and the power splitter and combiner have an insertion loss $1 / G_{S}$ and $1 / G_{C}$, respectively. Hence, that the receiver noise dominates, that is, no sky noise, the noise power at the input of the $i$ th (out of $M_{r}$ ) RCB is given by

$$
\begin{aligned}
N_{\text {Active }_{i}}= & \frac{G_{C_{i}}}{M_{s}} \sum_{j=1}^{M_{s}}\left(\left(N_{A}+N_{\mathrm{TL}_{j}}\right) \frac{G_{\mathrm{TL}_{j}} G_{L N A_{j}} G_{S_{j}} G_{\phi_{i j}}}{M_{r}}\right. \\
& +N_{\mathrm{LNA}_{j}} \frac{G_{\mathrm{LNA}_{j}} G_{S_{j}} G_{\phi_{i j}}}{M_{r}} \\
& \left.+N_{S_{j}} \frac{G_{S_{j}} G_{\phi_{i j}}}{M_{r}}+N_{\phi_{i j}} G_{\phi_{i j}}+N_{C_{i}}\right) \\
= & K T_{0} B_{\omega} \sum_{j=1}^{M_{s}}\left(\left(F_{\mathrm{LNA}_{j}} G_{\mathrm{LNA}_{j}}-1\right) \frac{G_{S_{j}} G_{\phi_{i j}} G_{C_{i}}}{M_{r}}+1\right),
\end{aligned}
$$

where $N_{S}, N_{\phi}$, and $N_{C}$ are the noise introduced by the power splitter, the phase shifter, and power combiner, respectively, where

$$
\begin{aligned}
& N_{S}=\left(\frac{M_{r}}{G_{S}}-1\right) K T_{0} B_{\omega}, \\
& N_{\phi}=\left(\frac{1}{G_{\phi}}-1\right) K T_{0} B_{\omega}, \\
& N_{C}=\left(\frac{1}{G_{C}}-1\right) K T_{0} B_{\omega},
\end{aligned}
$$

In (5), $G_{S}<1, G_{\phi}, G_{C}<1$ represent the power splitter gain-corresponding to the insertion loss-the phase shifter gain and the power combiner gain-corresponding to the insertion loss. Note that we have normalized (divided) the total noise power by the factor $M_{s}$ due to this assumption that the power divider is reciprocal, that is, $\left|S_{\text {io }}\right|^{2}=\left|S_{\mathrm{oi}}\right|^{2}=1 / M_{s}$ where the scattering parameters $S_{\mathrm{io}}$ and $S_{\mathrm{oi}}$ are associated with the $i$ th input port and the output port, and this fact that 


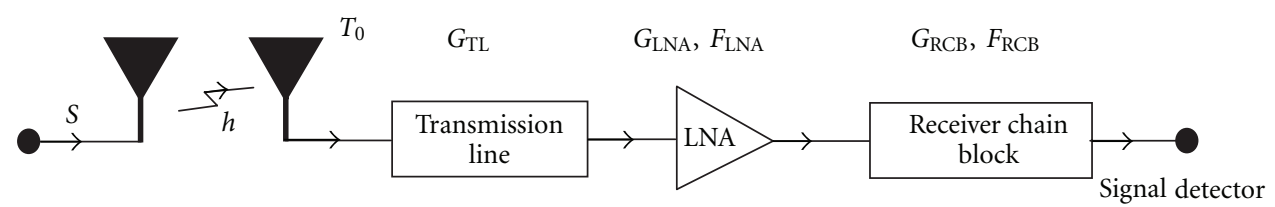

FIgURE 1: Single RF chain Transceiver.

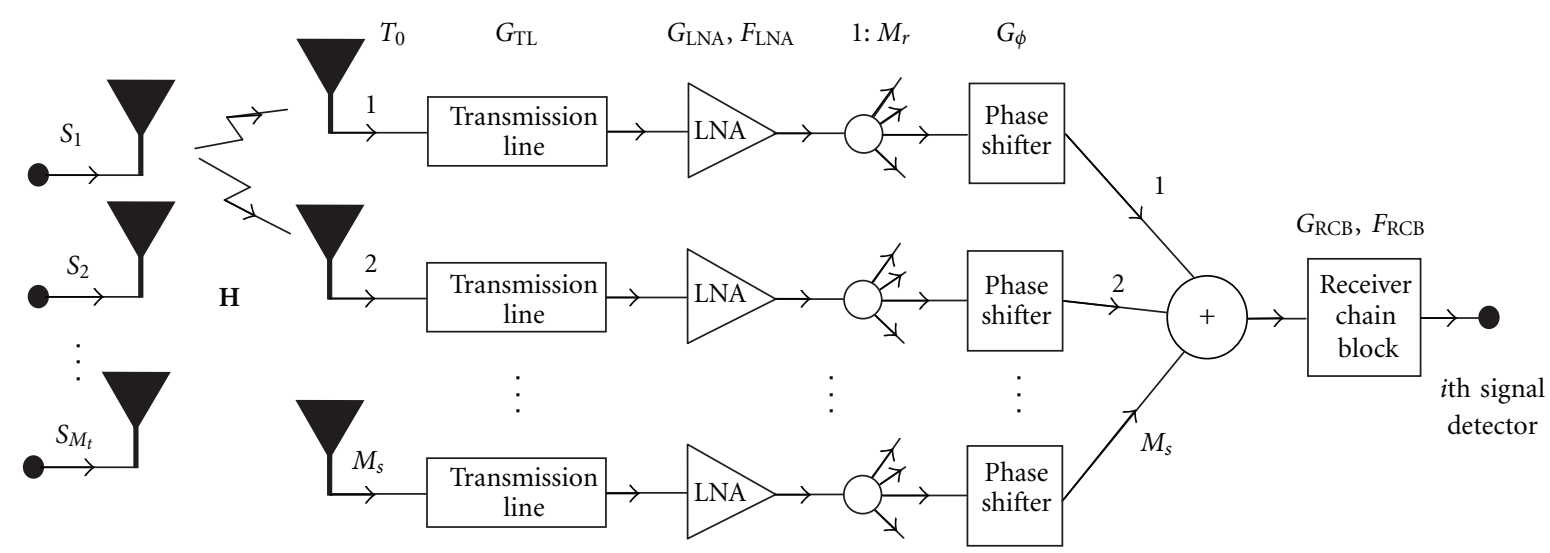

FIgURE 2: ith (out of $M_{r}$ ) receiver input for a SAS-MIMO with active phased array.

noise on each input branch is uncorrelated to the others [10, page 446].

The signal power is also given by

$$
S_{\text {Active }_{i}}=\frac{G_{C_{i}}}{M_{r} M_{s}} \sum_{j=1}^{M_{s}} \sum_{k=1}^{M_{t}}\left|\sqrt{G_{\mathrm{TL}_{j}} G_{\mathrm{LNA}_{j}} G_{S_{j}} G_{\phi_{i j}}} e^{j \phi_{i j}} h_{k j} s_{k}\right|^{2} .
$$

In (7), the signal power is also normalized to $M_{s}$ since the signal power at the combiner output is the summation of the powers of the input branches, that is, the law of conservation of energy [11]. Moreover, the phase shift components $\phi_{i j}$ are obtained form the optimum SAS-MIMO based on the spatial multiplexing/diversity transmission strategies proposed in [1]. Now, we perform the practical assumptions to simplify the above formulas in two steps.

(1) Assuming the phased array is uniform yields

$$
\begin{aligned}
N_{\text {Active }_{i}} & =K T_{0} B_{\omega}\left(\left(F_{\mathrm{LNA}} G_{\mathrm{LNA}}-1\right) \frac{G_{S} G_{\phi} G_{C}}{M_{r}}+1\right), \\
S_{\text {Active }_{i}} & =\frac{G_{C} G_{\mathrm{TL}} G_{\mathrm{LNA}} G_{S} G_{\phi}}{M_{r} M_{s}}\left|\sum_{j=1}^{M_{s}} \sum_{k=1}^{M_{t}} e^{j \phi_{i j}} h_{k j} s_{k}\right|^{2} .
\end{aligned}
$$

(2) Further, if we assume the ideal case $G_{\mathrm{TL}}=G_{C}=G_{\phi}=$ $G_{S}=1$ then

$$
\begin{aligned}
N_{\text {Active }_{i}} & =K T_{0} B_{\omega}\left(\left(F_{\mathrm{LNA}} G_{\mathrm{LNA}}-1\right) \frac{1}{M_{r}}+1\right), \\
S_{\text {Active }_{i}} & =\frac{G_{\mathrm{LNA}}}{M_{r} M_{s}}\left|\sum_{j=1}^{M_{s}} \sum_{k=1}^{M_{t}} e^{j \phi_{i j}} h_{k j} s_{k}\right|^{2} .
\end{aligned}
$$

Thus, SNR at the ith signal detector input is given by

$$
\operatorname{SNR}_{\text {Active }_{i}}=\frac{S_{\text {Active }_{i}}}{N_{\text {Active }_{i}}},
$$

where $S_{\text {Active }_{i}}$ and $N_{\text {Active }_{i}}$ are obtained based on one of abovementioned assumptions' results (8) and (9).

\section{Ray-Tracing Simulation}

The performance of the SAS-MIMO system is studied via a ray-tracing simulation in this chapter. There exist many ray-tracing tools for the deterministic modeling of the waves propagation environments. Among them, Radiowave Propagation Simulator (RPS) [12] has been shown to be accurate in terms of statistical properties and is widely used for the wave propagation simulations. The student version of this software is free for public.

3.1. Propagation Environment. In this paper, we employ a Three-Dimensional (3D) ray-tracing modeling to assess the signal coverage for a $2.4 \mathrm{GHz}$ wireless communication example. The Intelligent Multi-Antenna Radio Systems (iMARS) laboratory located in the third floor map of the EIT building at University of Waterloo is used as a typical indoor wireless environment (see Figure 3). This laboratory is structured by different wall materials including concrete and wood and furnished with tables, chairs, and shelves mostly built by wooden and plastic materials. Moreover, there exist various electronic equipments like computers, printers, and test devices in this lab. The reflection coefficients for the employed materials in $2.4 \mathrm{GHz}$ operating frequency are reported in [13-15]. Moreover, to evaluate the human body shadowing effect we use the measured permittivity data 



FIGURE 3: The simulated environment: A 2D top view of the third floor map of EIT building, University of Waterloo.

TABLE 2: Measured permittivity of indoor materials at $2.4 \mathrm{GHz}$.

\begin{tabular}{lcc}
\hline Type & $\begin{array}{c}\text { Thickness } \\
(\mathrm{mm})\end{array}$ & Complex $\epsilon_{r}$ \\
\hline Plexiglass I & 2.5 & $2.5-j 0.0235$ \\
Plexiglass II & 7.1 & $2.74-j 8.76 \times 10^{-4}$ \\
Blinds (closed) & 0.5 & $3.49-j 2.08 \times 10^{-4}$ \\
Blinds (open) & 22 & $1.96-j 1.1682 \times 10^{-4}$ \\
Red brick & 102 & $5.821-j 0.6753$ \\
Carpet & 7.75 & $1.32-j 7.8672 \times 10^{-5}$ \\
Fabric & 1.13 & $1.49-j 8.8804 \times 10^{-5}$ \\
Fiberglass & 890 & $1.02-j 9.3942 \times 10^{-4}$ \\
Glass & 2.5 & $6.3778-j 0.1659$ \\
Drywall I & 12.8 & $2.1899-j 0.0243$ \\
Drywall II & 9 & $2.49-j 0.0105$ \\
Linoleum & 1.61 & $3.08-j 0.0043$ \\
Fir & 37.7 & $2.5299-j 0.506$ \\
Particle-board & 19 & $2.6838-j 0.2953$ \\
Plywood & 18.5 & $2.4503-j 0.3111$ \\
Stucco & 25.75 & $0.9833-j 0.4219$ \\
Tiles & 21.2 & $3.0747-j 0.1807$ \\
Light cover & 2.5 & $1.66-j 0.0115$ \\
Tar paper & 1.7 & $2.4682-j 0.0953$ \\
Human skin & 5 & $39.5161-j 6.2029$ \\
\hline
\end{tabular}

for human skin in [16]. Table 2 summarizes the measured permittivity data at $2.4 \mathrm{GHz}$ used in this paper.

3.2. Wireless Communication Link Settings. In our simulation study, the transmitter antenna array is located at the corner of Hallway 1 and 2 (see Figure 3). The transmitter array consists of 4 patch antenna elements facing toward the iMARS lab
( $45^{\circ}$ rotation relative to Hallway 1 and 2 ), facing down with a small tilt of $8^{\circ}$, and $20 \mathrm{~cm}$ off from the ceiling. Each of transmitter elements has $0.1 \mathrm{~mW}$ transmitting power. A nominal patch beam pattern added to Figure 3 to demonstrate the transmitter antenna orientation. The patch antenna employed for the transmitter has a gain of $5.5 \mathrm{~dB}$. We have considered an array spacing $\lambda / 2$ for the transmitter. The receiver antenna array consists of an 8-element array of dipoles $(\lambda / 2$ length) with $0.1 \lambda$ element spacing located in the iMARS lab. We assume no mutual coupling as it could be considered as a part of channel and has no effect in our SNR calculations and comparisons. The dipole antenna employed for the receiver has a gain of $2 \mathrm{~dB}$. These array elements are chosen from a line of receiving points from locations $\mathrm{A}$ to $\mathrm{B}$ as shown in Figure 3. The antenna array moving steps are $5 \mathrm{~mm}$ apart through the line of receiving points which are at $1 \mathrm{~m}$ height from the lab floor. To simulate the shadowing effect, a human-body blocks the LOS path between the transmitter and the receiver.

3.3. System Model Definition. The different systems are compared based on a standard receiver settings. In this paper, we use WLAN 802.11 b standard. Settings for a typical 802.11 b receiver is given in Table 3. Moreover, to implement the SAS module we use the components shown in Table 1.

3.4. Simulation Results. As stated before, we perform the simulations on a 802.11 b (direct sequence WiFi) system architecture for all of under the test configurations. Assume this system provides a receiver sensitivity of $-76 \mathrm{dBm}$ (see Table 3 ). Starting with the $-174 \mathrm{dBm} / \mathrm{Hz}$ background thermal noise and adding $70 \mathrm{~dB}$ corresponding to the $11 \mathrm{MHz}$ channel bandwidth we obtain $-104 \mathrm{dBm}$ for the antenna noise. Subtracting this number from the required $-76 \mathrm{dBm}$ receiver sensitivity (minimum antenna signal), we calculate an input $\mathrm{SNR}$ of $28 \mathrm{~dB}$. If we assume the transmission 
TABLE 3: A typical receiver for WLAN 802.11 b standard [17].

\begin{tabular}{lc}
\hline Operating frequency & $2.4 \mathrm{GHz}$ \\
Bandwidth & $11 \mathrm{MHz}$ \\
Receiver sensitivity & $-76 \mathrm{dBm}$ \\
Total noise figure & $8.3 \mathrm{~dB}$ \\
Transmission line gain $G_{\mathrm{TL}}$ & $-1.7 \mathrm{~dB}$ \\
LNA gain $G_{\mathrm{LNA}}$ & $20 \mathrm{~dB}$ \\
LNA noise figure $F_{\mathrm{LNA}}$ & $3.5 \mathrm{~dB}$ \\
\hline
\end{tabular}
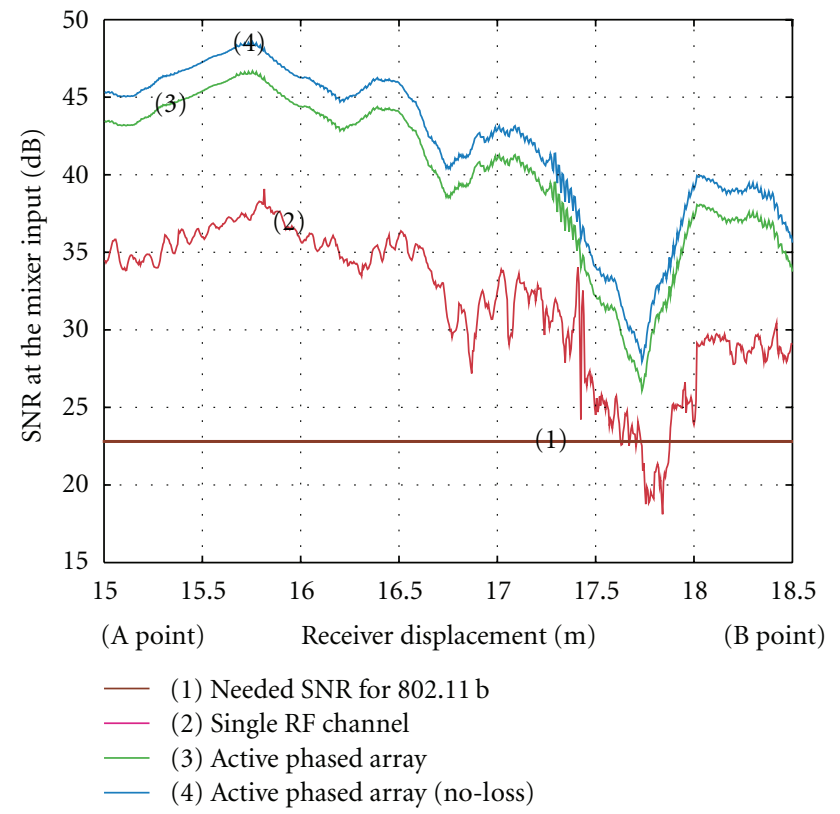

FIgURE 4: Provided SNR by SAS-MIMO from A to B points.

line TL (see Figure 1), including the filters and duplexer, has a maximum $1.7 \mathrm{~dB}$ noise figure (see Table 3 ), LNA provides $3.5 \mathrm{~dB}$ noise figure and everything else being ideal, a minimum $22.8 \mathrm{~dB}$ SNR is needed at the RCB input.

To show the effect of the SAS processing on the signal coverage, SNR at the RCB input along the path A-B for different architectures are shown in Figure 4. In this study, we assume that the same receiver electronic components are used for all of configurations. As the figure shows, a fullcomplexity MIMO even fails to receive signal in a deepfading areas like human body shadowing. However, when an active/passive array is used in the front end, due to beamforming capability the received power can be improved even $10 \mathrm{~dB}$. The post-SAS method gives the receiver an opportunity to receive the power even in a deep fading shown in the plot even with the loss in the implemented hybrid antenna selection network.

\section{Conclusion}

A receiver dynamic range analysis is performed for the post-LNA HAS to investigate on the provided SNR to the baseband. We have employed an operational WLAN
(802.11 b) system settings applied on an exact ray-tracing channel model. The results for a case study show that although the optimum post-LNA SAS works like a fullcomplexity MIMO in spatial multiplexing/diversity transmission strategies, it provides even a better SNR to the baseband. This issue becomes more important when a deep fading occurs and a full-complexity MIMO built by the regular receiver components, that is, $802.11 \mathrm{~b}$ here in this paper, cannot afford the fading.

\section{References}

[1] X. Zhang, A. F. Molisch, and S.-Y. Kung, "Variable-phase-shiftbased RF-baseband codesign for MIMO antenna selection," IEEE Transactions on Signal Processing, vol. 53, no. 11, pp. 4091-4103, 2005.

[2] P. Sudarshan, N. B. Mehta, A. F. Molisch, and J. Zhang, "Channel statistics-based RF pre-processing with antenna selection," IEEE Transactions on Wireless Communications, vol. 5, no. 12, pp. 3501-3510, 2006.

[3] J. Ahmadi-Shokouh, S. H. Jamali, and S. Safavi-Naeini, "Optimal receive soft antenna selection for MIMO interference channels," IEEE Transactions on Wireless Communications, vol. 8, no. 12, pp. 5893-5903, 2009.

[4] J. Ahmadi-Shokouh, S. H. Jamali, and S. Safavi-Naeini, "Pre-LNA smart soft antenna selection for MIMO spatial multiplexing/ diversity system when amplifier/sky noise dominates," European Transactions on Telecommunications, vol. 21, no. 7, pp. 663-677, 2010.

[5] J. Ahmadi-Shokouh, Receive soft antenna selection for noiselimited/interference MIMO channels, Ph.D. thesis, University of Waterloo, 2008.

[6] R. Vaughan and J. B. Andersen, Channels, Propagation and Antennas or Mobile Communications, IEE, London, UK, 2003.

[7] "Digital Control 5-Bit Phase shifter," part no. RFPSHT0204D RF-LAMBDA, http://www.rflambda.com/.

[8] "SMA Power Divider 4-Way 2-4 GHz," part no. MP8204-4 S. M. Electronics L.L.C., http://www.fairviewmicrowave.com/.

[9] "SMA Power Divider 8-Way 0.8-2.5 GHz," part no. MP8209-8 S. M. Electronics L.L.C., http://www.fairviewmicrowave.com/.

[10] A. Bahattacharyya, Phased Array Antennas, John Wiley \& Sons, Hoboken, NJ, USA, 2006.

[11] J. J. Lee, "G/T and noise figure of active array antennas," IEEE Transactions on Antennas and Propagation, vol. 41, no. 2, pp. 241-244, 1993.

[12] "Radiowave Propagation Simulator (RPS)," Radioplan, http:// www.radioplan.com/.

[13] R. Wilson, Propagation Losses Through Common Building Materials 2.4 GHz vs $5 \mathrm{GHz}$, Magic Networks, 2002.

[14] J. Jemai, R. Piesiewicz, and T. Krner, "Calibration of an indoor radio propagation prediction model at $2.4 \mathrm{GHz}$ by measurements of the IEEE 802.11 b preamble," in Proceedings of the IEEE 61st Vehicular Technology Conference, pp. 111-115, Spring 2005.

[15] T. Hult and A. Mohammed, "Assessment of multipath propagation for a $2.4 \mathrm{GHz}$ short-range wireless communication system," in Proceedings of the IEEE 65th Vehicular Technology Conference, pp. 544-548, April 2007.

[16] L. Roelens, W. Joseph, and L. Martens, "Comparison of path loss near homogeneous medium, layered medium and anatomically correct human model," in Proceedings of the IEEE 
Antennas and Propagation Society International Symposium, pp. 672-675, July 2006.

[17] G. Andrijevic, H. Magnusson, A. Kämpe, and H. Olsson, "Multistandard receiver for home networking and digital media," in Proceedings of the 22nd IEEE Norchip Conference, pp. 131-134, November 2004. 

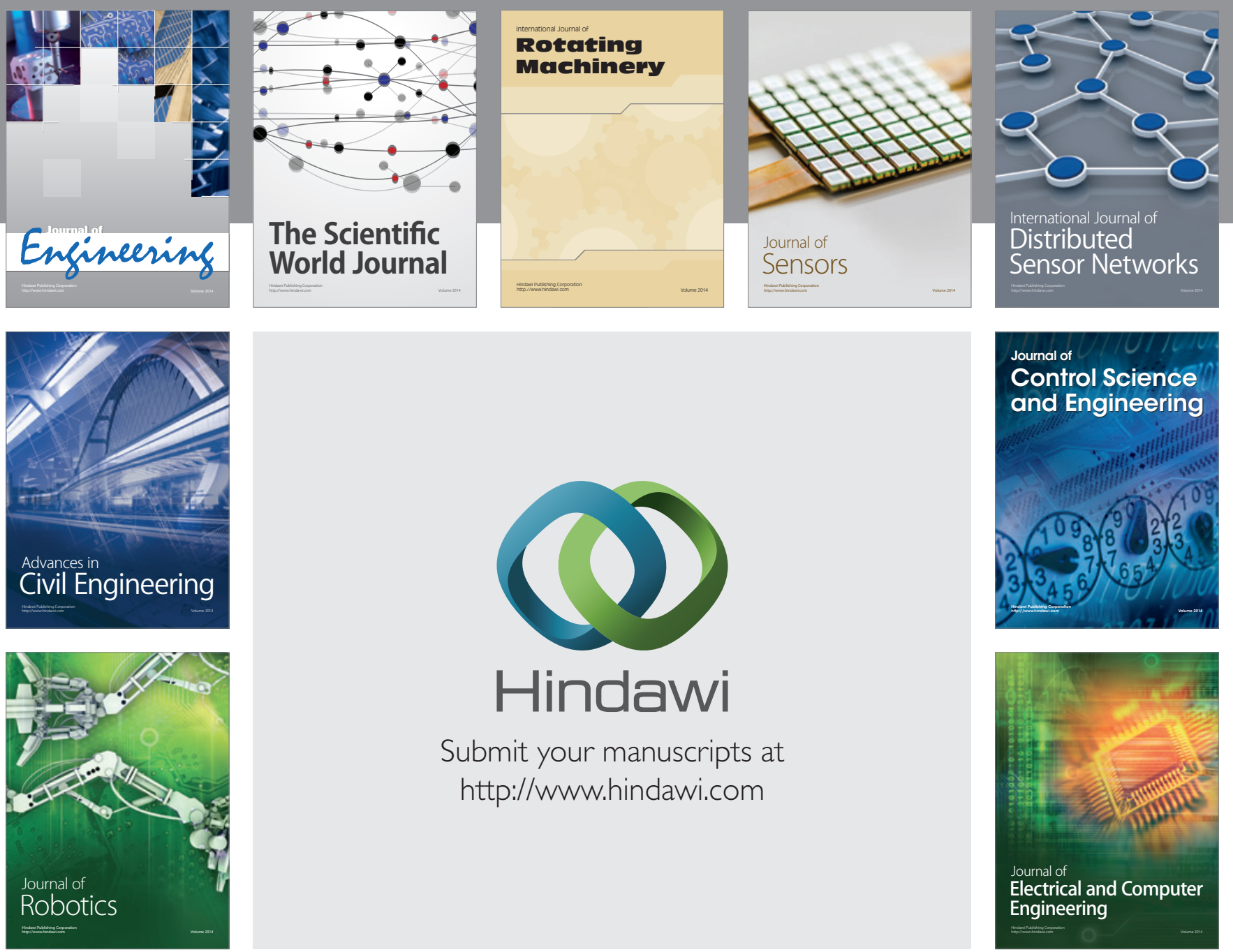

Submit your manuscripts at

http://www.hindawi.com
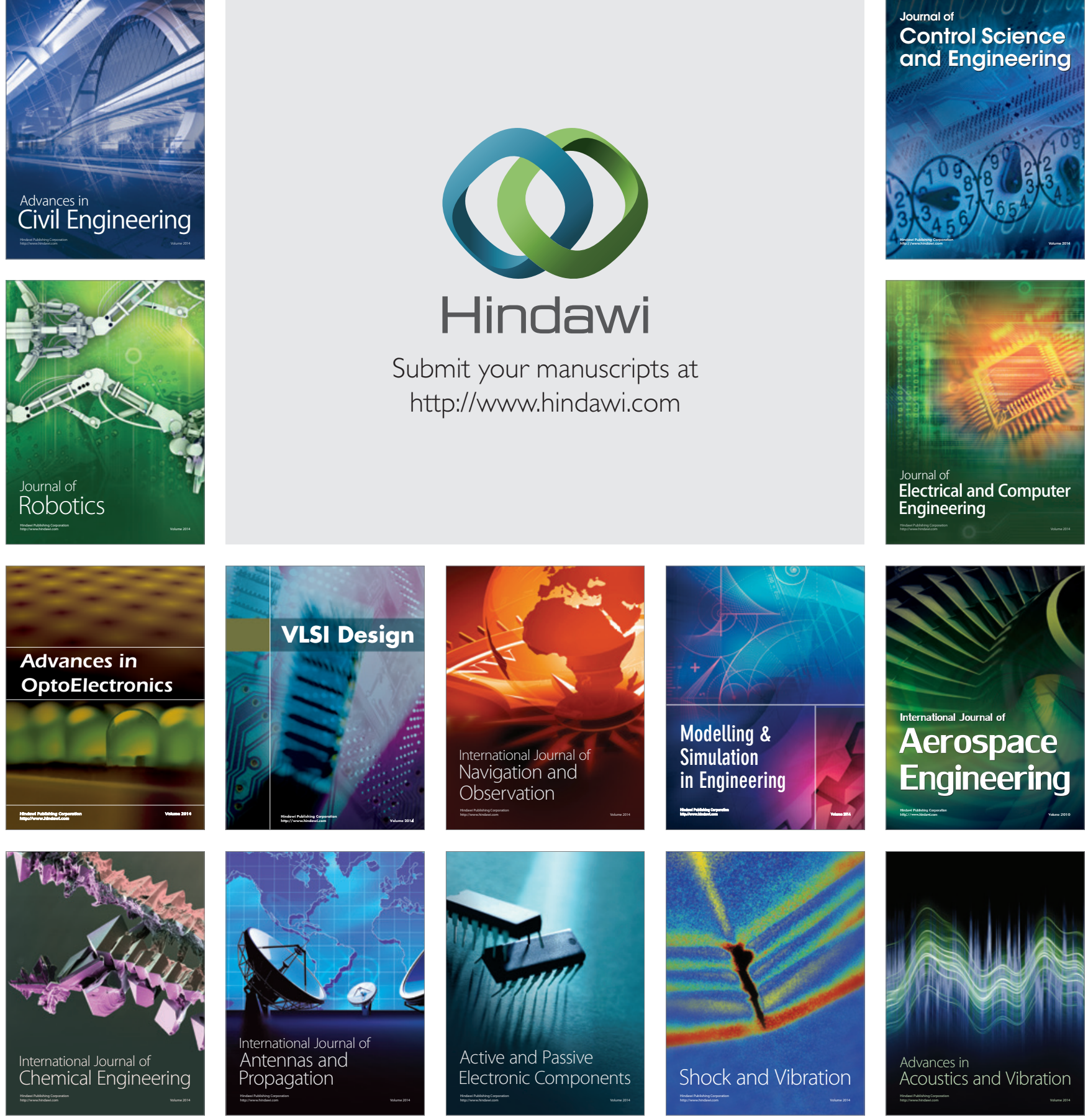